\title{
A sensitive resonance Rayleigh scattering sensor for dopamine in urine using upconversion nanoparticles
}

\author{
José Antonio Murillo Pulgarín (i) | Aurelia Alañón Molina (i) | Elisa Jiménez García | \\ Lucía García Gómez
}

Department of Analytical Chemistry and Foods Technology, University of CastillaLa Mancha, 13071 Ciudad Real, Spain

\section{Correspondence}

Aurelia Alañón Molina, Department of Analytical Chemistry and Foods

Technology, University of Castilla-La

Mancha, 13071 Ciudad Real, Spain.

Email: aurelia.alanon@uclm.es

\begin{abstract}
A highly sensitive resonance light scattering method for detecting dopamine in urine was developed by using a novel probe based on lanthanide-doped upconversion nanoparticles (UCNPs) linked to dopamine-quinone (DQ) by hydrogen bonding and electrostatic interactions. Adding dopamine to a solution containing UCNPs decreases their size and the intensity of their resonance light scattering signals. Based on the decrease, dopamine can be determined in Tris- $\mathrm{HCl}$ buffer and in urine samples spiked with analyte concentrations over the range $0-300 \mu \mathrm{M}$ with a limit of detection of $1.62 \mu \mathrm{M}$. As-prepared UD can thus provide an effective platform for biosensor development, drug discovery, and rapid diagnosis of Parkinson's and Alzheimer's disease, among other medical conditions.
\end{abstract}

\section{KEYWORDS}

dopamine, lanthanide, nanoparticles, resonance Rayleigh scattering, urine

\section{1 | INTRODUCTION}

Dopamine, a catecholamine compound, is a neurotransmitter that manages a wide range of cognitive functions including motivation, behaviour, learning, and memory. ${ }^{[1-3]}$ The concentration of dopamine (DA) in biological fluids such as blood plasma, urine, and extracellular fluid in the central nervous system influences human health and can be used as an indicator of various diseases. ${ }^{[4,5]}$ Thus, redundant secretion of DA by effect of, for example, Huntington's disease, is associated with failure in energy metabolism and can ultimately lead to death. By contrast, a lack of DA prevents muscle control and can cause distraction of even Parkinson's disease. ${ }^{[6]}$ The relationship between this compound and human pathologies has been known for more than 150 years. ${ }^{[7]}$ Actually, many researchers are interested in the function and measurement of catecholamine neurotransmitters, including DA, as they have the potential to serve as clinically relevant biomarkers for specific disease states or to monitor treatment efficacy. Neurotransmitters are present throughout the body, and measurements of their concentration levels can be performed in various biological fluids, including urine. A need therefore exists for effective methods to measure DA in biological fluids with a view to accurately diagnosing some diseases. ${ }^{[8,9]}$ A number of methods based on electrochemical analysis, ${ }^{[10,11]} \mathrm{UV}-\mathrm{Vis}$ spectrometry, ${ }^{[12]}$ high-performance liquid chromatography, ${ }^{[13]}$ and fluorescent probes ${ }^{[14,15]}$ have been developed for this purpose. Although these methods can offer good selectivity and low limits of detection, they are sometimes inappropriate for routine analysis. For example, ascorbic acid usually coexists with DA in large excess in biological samples, and it is easily oxidized at the potential close to those required for the oxidation of catecholamines; due to this, its oxidation currents dominate the electrochemical signal. Moreover, the oxidized DA can catalyse the oxidation of ascorbic acid. This leads to serious interference in the determination of DA by electrochemical methods. ${ }^{[16]}$ Another critical problem associated with the detection of 
DA is the passivation of the electrode surface caused by the polymeric melanin type of oxidation products of DA. On the other hand, chromatographic methods are expensive, often have long analysis time, and complicate pretreatment. Only few studies described methods with no cleanup of the urine sample. However, these methods made use of different precolumn derivatization procedures, which means that is not shorter than methods using a clean-up procedure. An easier, more cost-effective alternative is thus needed to efficiently determine DA.

The special properties of nanomaterials in relation to bulk materials have aroused growing interest in their use. The high activity, good selectivity, and vast specific surface of nanomaterials have promoted extensive research into their biosensor design potential. Metal nanoparticles are especially outstanding in this respect. Rare-earth-doped upconversion nanoparticles (UCNPs) emitting higher energy visible light upon excitation with low-energy nearinfrared (NIR), which were developed in the 1980s, have recently aroused considerable attention. ${ }^{[17]}$ The unique luminescence mechanism of UCNPs has several advantages over conventional fluorescent labels including improved detection sensitivity by effect of the absence of autofluorescence, minimal photodamage to living organisms due to their easier penetration by NIR light, good chemical and physical stability, and low toxicity. ${ }^{[18]}$

Resonance light scattering (RLS) is a relatively new spectral analysis technique, which has received much attention because it was originally proposed by Pasternack et al. ${ }^{[19]}$ in 1993. RLS signals are obtained by simultaneously scanning the excitation and emission monochromators of a spectrofluorometer. The high sensitivity and convenient operation of the RLS technique have made it popular for quantifying various analytes. RLS has in fact emerged as a highly attractive choice for monitoring molecular assemblies and characterizing extended aggregates of chromophores. In recent years, it has also been used to determine drugs ${ }^{[20]}$ and various biological macromolecules such as nucleic acids ${ }^{[21,22]}$ and $\mathrm{H}_{2} \mathrm{~S} .^{[23]}$

In this work, we developed a DA sensor using upconversion nanoparticles as an RLS probe. Strong interactions between DA and UCNPs decrease the size of the nanoparticles and their RLS signals. The sensor was used to determine trace concentrations of DA in biological samples with acceptable results.

\section{2 | EXPERIMENTAL}

\section{1 | Reagents}

All experiments were performed with analytical reagentgrade chemicals and pure solvents, and all solutions were prepared using ultrapure distilled water with a total organic carbon level $<5 \mu \mathrm{g} \mathrm{L}{ }^{-1}$, which was obtained from a Milli-Q 185 plus system. Hydrochloric acid $37 \%$ and ethanol 96\% v/v were supplied by Panreac Química S.A. (Barcelona, Spain); sodium fluoride and yttrium(III) nitrate pentahydrate were obtained from Merck (Darmstadt, Germany). Ytterbium(III) nitrate pentahydrate, erbium(III) nitrate pentahydrate, poly (acrylic acid) (PAA), tris(hydroxymethyl)aminomethane (Tris-HCl), and DA hydrochloride were provided by Sigma Aldrich (St. Louis, USA).

The PAA stock solution $\left(50 \mathrm{~g} \mathrm{~L}^{-1}\right)$ was prepared by dissolving exactly $12.50 \mathrm{~g}$ of the chemical in a mixed solvent ethanol-water (1:1), transferring this solution to a 250-ml calibrated flask and filling to the mark with the same mixed solvent. Sodium fluoride stock solution (11.67 $\mathrm{g} \mathrm{L}^{-1}$ ) was prepared at the same way as previous, dissolving $2.92 \mathrm{~g}$ of the chemical in a mixed solvent ethanol-water (1:1) and transferring this solution to a 250-ml calibrated flask.

The UCNPs cannot be dissolved, so a suspension of them are prepared in a $10-\mathrm{ml}$ volumetric flask, weighing exactly $12 \mathrm{mg}$ and bringing to the indicated volume with Tris- $\mathrm{HCl}$ buffer. This solution was found to be stable thanks to stability studies also described in this article for at least a several months and was stored in the dark at $4^{\circ} \mathrm{C}$.

DA hydrochloride working solution $\left(1.5 \mathrm{mmol} \mathrm{L}^{-1}\right)$ was prepared by dissolving exactly to the volumetric mark of $2.84 \mathrm{mg}$ of the chemical in a 10-ml volumetric flask with Tris- $\mathrm{HCl}$ buffer $\left(0.5 \mathrm{~mol} \mathrm{~L}^{-1}, \mathrm{pH}=8.5\right)$. This solution is easily oxidized, so it is necessary to prepare it by knowing the time of exposure to the environment because DA tends to turn dark brown at slightly basic $\mathrm{pH}$ with the presence of oxygen, giving rise to melanin. So the solution described above was prepared daily and was kept protected from light and at a temperature of $4^{\circ}$ $\mathrm{C}$ until it is used.

Urine samples were collected early from fasting healthy people. Following centrifugation for $15 \mathrm{~min}$, the supernatant was diluted 1:10 with water and maintained at $4^{\circ} \mathrm{C}$ to improve stability.

\section{2 | Apparatus}

All fluorometric measurements were made on an AmincoBowman Series 2 Luminescence Spectrometer governed via the software AB2 for the OS2 operating system. The instrument was equipped with a flash 7-W xenon lamp, Czerny-Turner monochromators with a focal distance of $200 \mathrm{~mm}$, and holographic gratings with 1,200 lines $\mathrm{mm}^{-1}$ adjustable slits on both monochromators and a gated 
photomultiplier. Quartz glass cuvettes of $1.0 \mathrm{~cm} \times 1.0 \mathrm{~cm}$ light path were used.

All spectral measurement and treatment of data were carried out with a Beckman Instrument DU-70 Spectrophotometer connected to computer fitted with Beckman Data Leader Software.

For pH measurements, a Crison $2001 \mathrm{pH}$ meter was used. A Selecta thermostatic bath was coupled to the cuvette holder for the temperature control of the working solutions. Selecta Mixtaxel centrifuge was used in the preparation of the solutions.

\section{3 | Software}

The software Ftotal ${ }^{[24]}$ was used to obtain a total RLS spectrum. Three-dimensional spectra can be obtained and presented as contour maps. The Ftotal program not only generates information through the isometric representation of a level curve but also processes the spectral data to obtain any type of bidimensional spectrum.

\section{4 | Preparation of PAA-modified UCNPs}

Water-soluble PAA-UCNPs were prepared by using a one-pot hydrothermal method described elsewhere. ${ }^{[25,26]}$ In brief, to acquire $\mathrm{Ln}\left(\mathrm{NO}_{3}\right)_{3}(\mathrm{Ln}=\mathrm{Y}, \mathrm{Yb}, \mathrm{Er}), 0.25 \mathrm{mmol}$ of a 0.78:0.2:0.02 mixture of $\mathrm{Y}, \mathrm{Yb}$, and Er lanthanide oxides was dissolved in nitric acid at $65^{\circ} \mathrm{C}$, the acid being evaporated after $6 \mathrm{~h}$ of reaction. The as-obtained nitrate salts were added to a solution containing $900 \mathrm{mg}$ of PAA and supplied with another aqueous solution containing $0.21 \mathrm{~g}$ of $\mathrm{NaF}$ added dropwise under vigorous stirring. Both PAA and NaF were dissolved in 1:1 ethanol-water. Then, the mixture was transferred into a 50-ml Teflon autoclave and heated at $180^{\circ} \mathrm{C}$ for $24 \mathrm{~h}$ (hydrothermal treatment). A precipitate was obtained after allowing the Teflon autoclave to cool to room temperature, and then the precipitate was centrifuged, and washed with ethanol and water several times, and dried under vacuum before use. A solution of this product in water at $\mathrm{pH} 8.5$ adjusted with Tris- $\mathrm{HCl}$ buffer was found to remain stable for at least 3 days.

\section{5 | General procedure}

For DA detection, an aqueous solution containing a concentration of $0.20 \mathrm{mg} \mathrm{ml}^{-1}$ of as-prepared of UCNPs and a variable concentration of DA was added to the quartz cuvette. The $\mathrm{pH}$ and reaction time were optimized in additional tests. The final volume of all samples was
$1 \mathrm{ml}$, and the concentration of UCNPs was $0.20 \mathrm{mg} \mathrm{ml}^{-1}$ in all tests.

Samples were scanned over the spectral range of $10,000-50,000 \mathrm{~cm}^{-1}$ at $h=1 / 2$.

The intensity of the RLS spectra was measured at $33,333.3 \mathrm{~cm}^{-1}$ of the UD system. Calibration was based on the change in $\log \left(I_{0} / I\right)$ as a function of the DA concentration, with $I$ and $I_{0}$ being the intensity in the presence and absence of analyte. The calibration graph was run from three replicates per point.

\section{6 | Urine samples}

Human urine was collected from healthy adult volunteers and centrifuged for $10 \mathrm{~min}$ to remove solids. All supernatants were diluted 625-fold before analysis and spiked with different concentrations of DA. The actual amount of analyte present in each sample was determined by following the general procedure. A $\log \left(I_{0} / I\right)$ versus DA concentration plot was constructed, and the unknown concentration calculated by using the standard addition method. Each determination was performed in triplicate.

\section{3 | RESULTS AND DISCUSSION}

\section{1 | Characterization of UCNPs}

UCNP morphology was characterized by transmission electron microscopy. As can be seen from the micrograph of Figure 1, mixing UCNPs with DA caused the latter to form a coating that increased in thickness with time (from about $4 \mathrm{~nm}$ after $30 \mathrm{~min}$ to about $10 \mathrm{~nm}$ after $90 \mathrm{~min})$.

\section{2 | Reaction of UCNPs with DA}

UCNPs and DA were linked by hydrogen bonding and electrostatic interactions that reduced leading RLS in the nanoparticles. At a given concentration of UCNPs (0.204 $\mathrm{mg} \mathrm{ml}^{-1}$ ), the RLS signal decreased gradually with increasing amount of DA and eventually disappeared at a concentration of $5 \mathrm{mM}$. The UV spectra of Figure 2 confirm that the DA-melanin ensemble linked to UCNPs (UD). Thus, the spectra included a new peak at $280 \mathrm{~nm}$ typical of DA. Also, they were consistent with the presence of $-\mathrm{COOH}$ groups on nanoparticle surfaces and $-\mathrm{NH}_{2}$ groups in molecular DA, as well as with the formation of $\mathrm{N}-\mathrm{H} \cdots \mathrm{O}$ and $\mathrm{O}-\mathrm{H} \cdots \mathrm{O}$ hydrogen bonds.

Incubating UCNPs with DA in Tris- $\mathrm{HCl}$ buffer at $\mathrm{pH}$ 8.5 caused the solution to switch from colourless to dark grey with time (Figure S1A), the process being monitored 


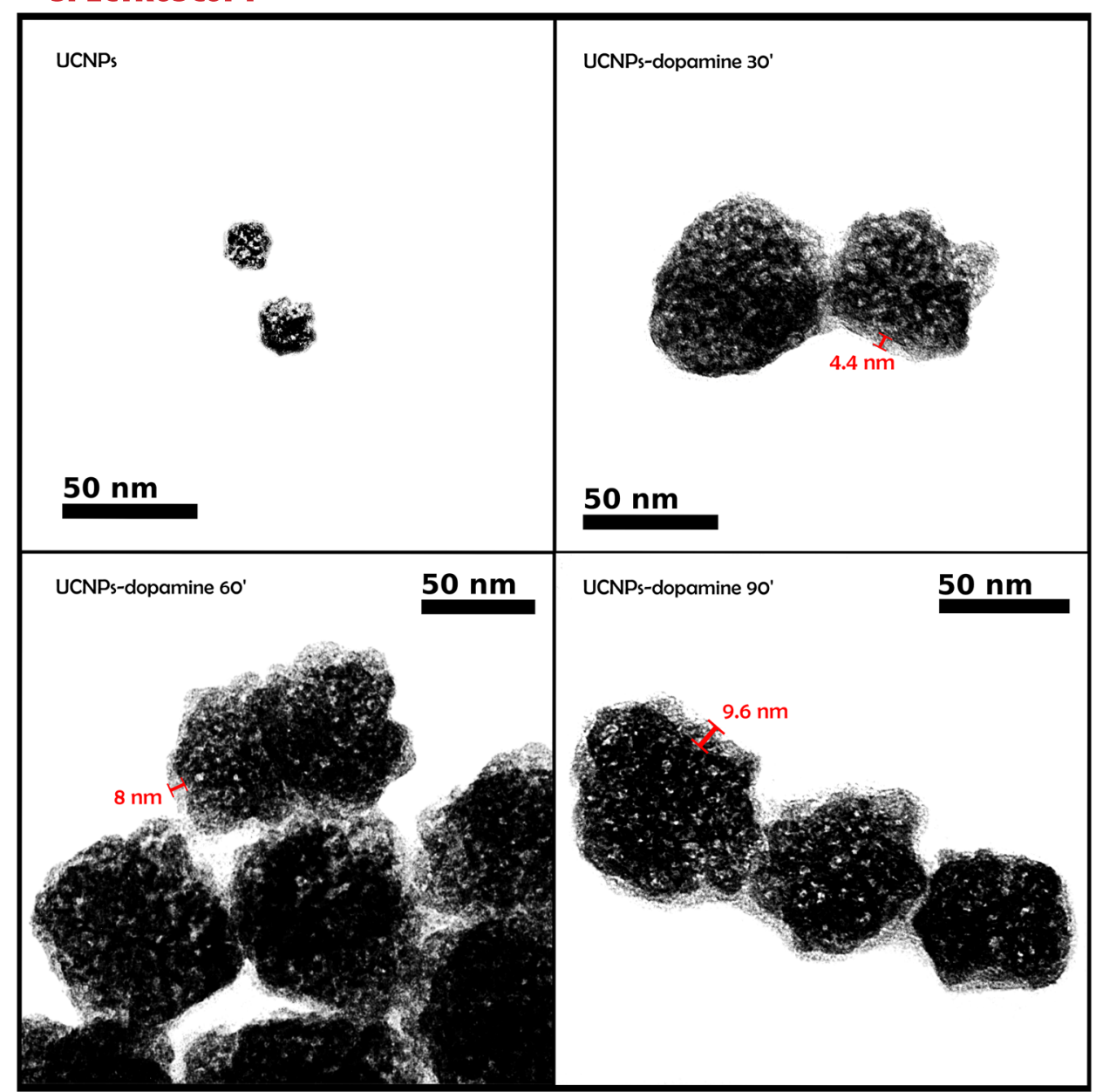

FIGURE 1 TEM images of the formation of dopamine-melanin nanoshells on the surface of UCNPs. The measurement was taken at the time points of $0,30,60$, and 90 min after the addition of dopamine to the UCNP solution. TEM, transmission electron microscopy; UCNPs, upconversion nanoparticles

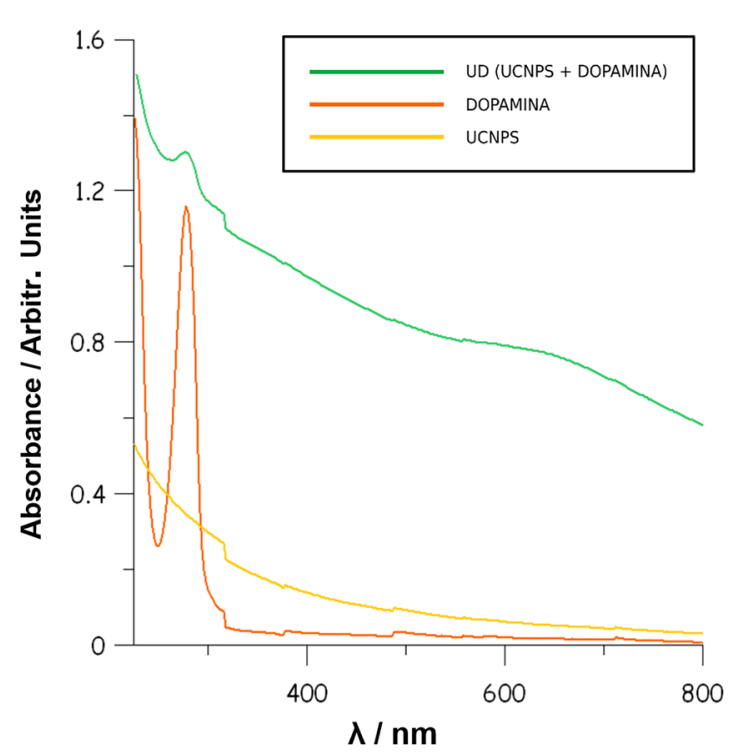

FIGURE 2 UV spectra of UCNPs, DA, and UD. DA, dopamine; UCNPs, upconversion nanoparticles by UV-Vis spectroscopy. The broad, monotonic absorbance band extending from the NIR to the UV region (Figure S1B) is typical of synthetic melanin. ${ }^{[27]}$ The absorbance increased with the reaction time, which is consistent with the formation of DA-melanin in the mixed solution. The colour change was accompanied by a strong decrease in RLS for the UCNPs throughout the reaction that was quantitatively measured (Figure S2).

\section{3 | Optimization of the experimental conditions}

The conditions for the sensing analysis were optimized by incubating different concentrations of DA with a fixed concentration of UCNPs at $\mathrm{pH}$ 8.5. The quenching effect of DA-melanin on the nanoparticles was concentration dependent below a DA concentration of $5 \mathrm{mM}$. A rapid decrease in the RLS signal was observed from 0 to $80 \mathrm{~min}$, which was followed by a plateau at longer 
reaction times. A DA concentration of $1 \mathrm{mM}$ per $0.2 \mathrm{mg} \mathrm{ml}^{-1}$ UCNPs and preincubation for $90 \mathrm{~min}$ were used to ensure a broad-enough linear detection range in subsequent detection tests.

RLS spectra were recorded and RLS intensities measured with a fluorescence spectrophotometer by simultaneously scanning the excitation and emission channels under the condition $\bar{v}_{\text {exc }}=\bar{v}_{\text {em }}$ (i.e., $\bar{v}_{\text {em }} / \bar{v}_{\text {exc }}=1$ ). The optimum $\bar{v}_{\text {em }} / \bar{v}_{\text {exc }}$ ratio was established from a threedimensional spectrum that was obtained by recording 61 excitation spectra with the emission wavelength in $9.6 \mathrm{~nm}$ steps from 10,799 to $28,571 \mathrm{~cm}^{-1}$, using a solution containing $0.2 \mathrm{mg} \mathrm{ml}^{-1}$ UCNPs and $100 \mu \mathrm{M}$ of DA at $\mathrm{pH}$ 8.5 (Figure 3). As can been seen, the excitation and emission branches both saturated at $21,052.6 \mathrm{~cm}^{-1}$. Also, an unsaturated peak was observed at $16,666.7 \mathrm{~cm}^{-1}$ of excitation and $33333.3 \mathrm{~cm}^{-1}$ of emission $\left(\bar{v}_{\text {em }} / \bar{v}_{\text {exc }}=1 / 2\right)$, and two weaker ones at an $\bar{v}_{\mathrm{em}} / \bar{v}_{\mathrm{exc}}$ ratio of $2 / 3$ and 2 , respectively.

These results led us to reconstruct the 3D spectrum, albeit at a lower sensitivity in order to avoid Rayleigh saturation. However, the new spectrum was not reproducible; in fact, it was rather uneven, which led us to return to the previous spectrum and to use a $v_{\text {em }}^{-} / v_{\text {exc }}^{-}$ ratio of $1 / 2$ (i.e., excitation at $33,333.3 \mathrm{~cm}^{-1}$ to obtain the peak at $300 \mathrm{~nm}$ in the emission spectrum as seen in Figure 4).

\section{4 | Calibration}

We used the above-described optimum conditions to develop a method for determining DA over the concentration range of $20-300 \mu \mathrm{M}$. The calibration graph was constructed from three replicate measurements. A linear

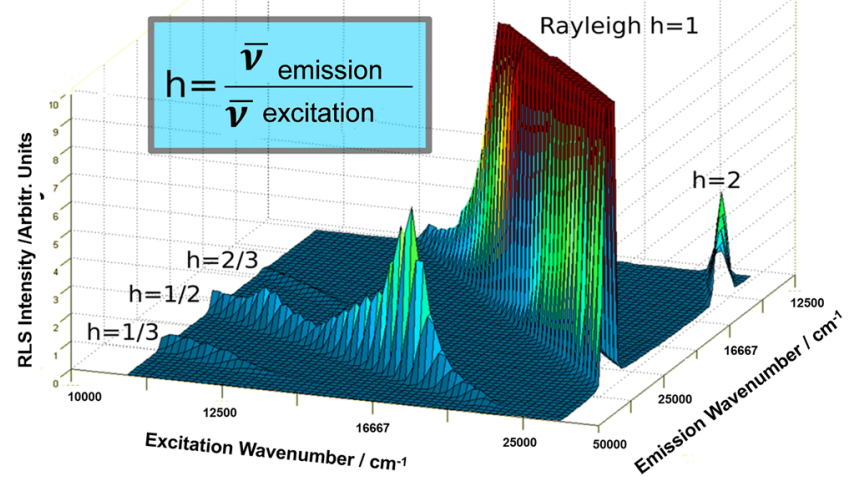

FIGURE 3 Three-dimensional spectrum of the UCNPs@dopamine-melanin hybrid system in a Tris-HCl buffer ( $\mathrm{pH}=8.5$ ). RLS, resonance light scattering; UCNPs, upconversion nanoparticles relationship between $\log \left(I_{0} / I\right)$ and $\log$ [dopamine] was observed. Figure 5 shows the RLS signal for one of the replicates at each concentration level.

The results of the proposed method were evaluated by fitting experimental data to a least squares line of the type $y=a+b x$ after discarding outliers by least median of squares (LMS) regression. ${ }^{[28]}$ LMS is a robust regression method capable of accurately detecting outliers that cause errors in the true line when experimental data are fitted by least squares regression. No outliers were detected here, however. Table 1 shows the results of the statistical analysis. As can been seen, both methods provided similar results, the coefficients of determination for least squares and LMS being 0.993 and 0.996 , respectively.

Representativeness in the calibration graph was maximized by overall least squares regression. The accuracy of the regression was checked via an analysis of variance (ANOVA) $)^{[29]}$ based on which the variance of the residual error did not change from sample to sample or among calibration graphs.

An ANOVA involves calculating the variance ratio (viz., the experimental $F$ value) and comparing it with the theoretical $F$ value for an adequate number of degrees of freedom at the $95 \%$ of confidence level. $F_{1}$ compares total deviations from the overall linear regression with the deviation within each set from the set line. The experimental value of $F_{1}$ was 3.22 and hence less than the theoretical value (3.26 at the $95 \%$ confidence level); consequently, the departure of individual sets from the overall regression line was not significant. $F_{2}$ compares differences among regression coefficients and slopes with the deviation within each set from the set line. The experimental value of $F_{2}$ was 2.41 , which is less than the theoretical value (3.89), so there were no significant differences among regression coefficients; and their mean, overall slope was adopted as the representative slope.

Residual analysis showed that the calibration line complied with homoscedasticity criteria. ANOVA was used to assess the regression line for linearity. ${ }^{[30]}$ As can be seen from Table 2, the experimental $F$ value was smaller than its theoretical counterpart. Therefore, the lack of fit of the term was not significant, and the model provided an acceptable description of the linear relationship between $\log \left(I_{0} / I\right)$ and $\log$ [dopamine].

\section{5 | Limit of detection, repeatability, and selectivity}

If error propagation is assumed, limits of detection and determination are representative of the reliability of blank measurements and signal measurements of 


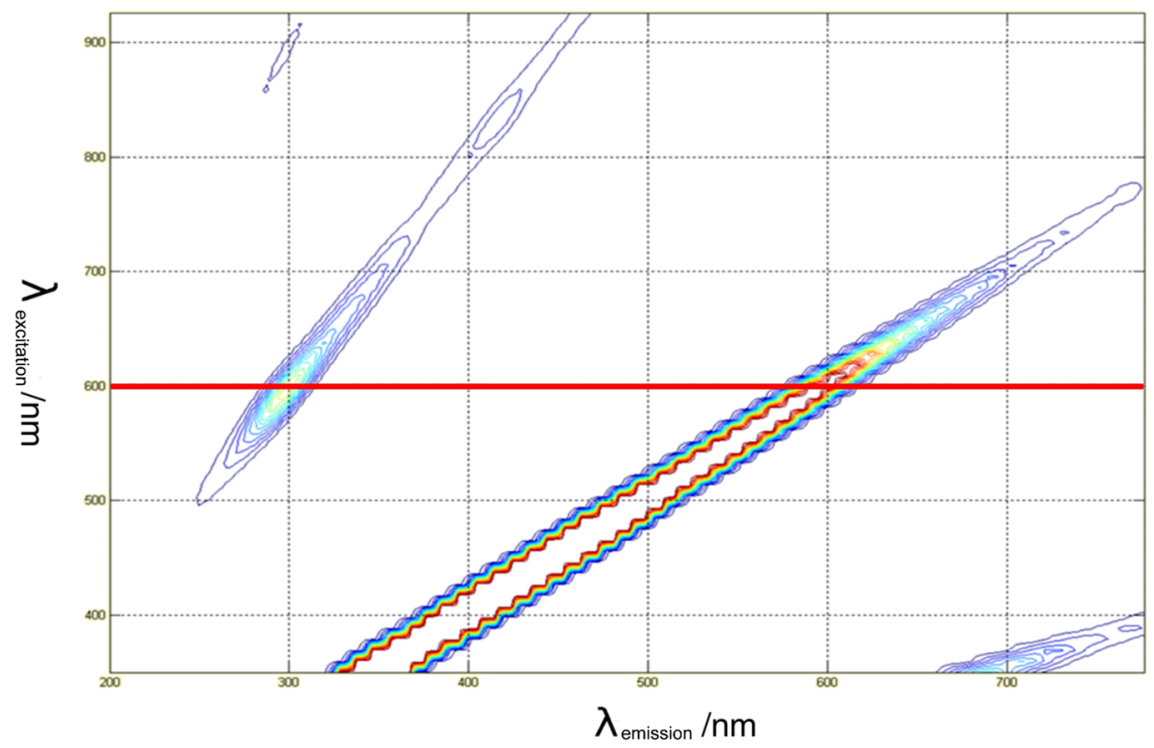

FIGURE 4 Total RLS spectrum of the UCNPs@dopamine-melanin hybrid system in a Tris- $\mathrm{HCl}$ buffer $(\mathrm{pH}=8.5)$ as contour map and the selected bidimensional spectrum. RLS, resonance light scattering; UCNPs, upconversion nanoparticles [Colour figure can be viewed at wileyonlinelibrary.com]

standards. ${ }^{[31,32]}$ Our limit of detection was $1.41 \mu \mathrm{M}$, but that calculated according to Clayton ${ }^{[33]}$ was $1.62 \mu \mathrm{M}$, the latter being the more suitable because, despite being its being higher, it provides for both false positives and false negatives $(\alpha=\beta=0.5 ; n-2=16$ ).

The repeatability of the proposed method was assessed by using 10 solutions containing $40.0 \mu \mathrm{M}$ of DA. The relative error was $1.05 \%$ as calculated according to IUPAC and $1.07 \%$ based on error propagation theory.

To assess the selectivity, the effect of ascorbic acid and uric acid was next evaluated. Different synthetic solutions, containing DA $(100 \mu \mathrm{M})$ and one of the compounds in variable proportions, were prepared. The maximum assayed proportion was $1 / 50$. The RLS signal of these

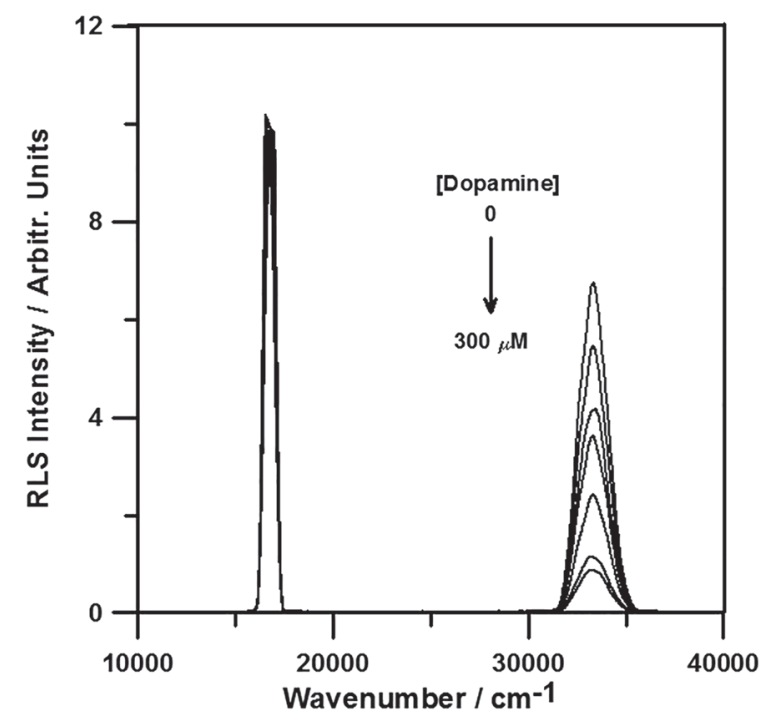

FIGURE 5 Evolution of RLS spectra of UCNPs with increasing concentration of dopamine from top to bottom (0, 20.0, 30.0, 40.0, 80.0, 200.0, and $300.0 \mu \mathrm{M}$, respectively). RLS, resonance light scattering; UCNPs, upconversion nanoparticles solutions was then measured using the developed method and optimized experimental conditions. The results of these experiences show that the presence of these compounds does not affect significantly the determination of DA, as well as the recovery percentages in each case.

\subsection{Determination of DA in urine}

In real-life diagnostic applications, the DA concentration in urine can directly reflect the activity of the sympathoadrenal system. DA in urine is important for diagnosing pheochromocytoma and related diseases. ${ }^{[7]}$ In this work, we used three human urine samples to assess the performance of the proposed method in

TABLE 1 Regression parameters obtained by a least squares fit and a least median of squares fit of the data, with three replicates per point

\begin{tabular}{|lc|}
\hline Parameter & Least squares \\
\hline Intercept, $a$ & -.81 \\
\hline Standard deviation of intercept, $s_{a}$ & .06 \\
\hline Slope, $b$ & .663 \\
\hline Standard deviation of slope, $s_{b}$ & .014 \\
\hline Standard deviation of regression, $s_{y x}$ & .025 \\
\hline Coefficient of determination, $R^{2}$ & .993 \\
\hline Parameter & \\
\hline Intercept, $a$ & Least median of squares \\
\hline Slope, $b$ & -.83 \\
\hline Standard deviation of regression, $s_{y x}$ & .76 \\
\hline Coefficient of determination, $R^{2}$ & .02 \\
\hline
\end{tabular}


TABLE 2 ANOVA test (linearity of the calibration graph)

\begin{tabular}{|cccccc|}
$\begin{array}{l}\text { Source of } \\
\text { variation }\end{array}$ & SS & $\boldsymbol{d} \boldsymbol{f}$ & $\mathbf{M S}$ & $\boldsymbol{F}_{\text {exp }}$ & $\boldsymbol{F}_{\text {theor }}$ \\
\hline $\begin{array}{l}\text { Due to regression } \\
\text { Set means about } \\
\text { line (lack of fit) }\end{array}$ & 1.454 & 1 & 1.454 & 1.72 & 3.26 \\
\hline $\begin{array}{l}\text { Within line } \\
\quad \text { pure error) }\end{array}$ & $6.524 \times 10^{-3}$ & 12 & $5.436 \times 10^{-4}$ & \\
\hline Total & 1.464 & 17 & $9.613 \times 10^{-2}$ & \\
\hline
\end{tabular}

Abbreviations: ANOVA, analysis of variance; $d f$, degrees of freedom; MS, mean squares; SS, sum of squares of deviation.

detecting DA in urine. For this purpose, a calibration curve was constructed by using the standard addition method owing to the strong matrix effect of urine. The method of standard additions is often the technique utilized when accurate quantitative results are desired, used in analytical analysis. This is often used when there are other components in the sample of interest that causes either a reduction or an enhancement of the signal desired for quantitative results. When this is the case, one cannot simply compare the analyte signal with standards using the traditional calibration curve approach. The operating procedure followed is described in Section 2. The method allowed DA in urine to be successfully determined with an average recovery of $103.0 \%$ and a standard deviation of only $2.0 \mu \mathrm{M}$.

The determination of DA was validated by using least squares regression ${ }^{[29]}$ to compare the results of the proposed method with those of a reference fluorimetric method. ${ }^{[34]}$ Both were used to analyse 10 samples containing the analyte at levels within the operating range. The concentrations obtained with both methods were subjected to least squares paired analysis, which considers the potential effects of various types of error. The presence of random of errors in the test method causes points to scatter around the least squares line, and the calculated slope and intercept to slightly depart from unity and zero, respectively, as a result. Random errors can be estimated from the standard deviation in the $y$-direction (also called "the standard deviation of the estimate of $y$ on $x$ ").

A proportional systematic error leads to a change in $b$, so the difference between $b$ and unity provides an estimate of the proportional error. A constant systematic error results in a nonzero intercept. If both methods provided identical concentrations in the same samples, then least squares analysis would give a zero intercept and a unity slope. Figure 6 shows the 95\% confidence region for the true slope and estimated intercept. As can be seen, the point corresponding to the zero intercept and unity slope falls within the joint confidence region, which

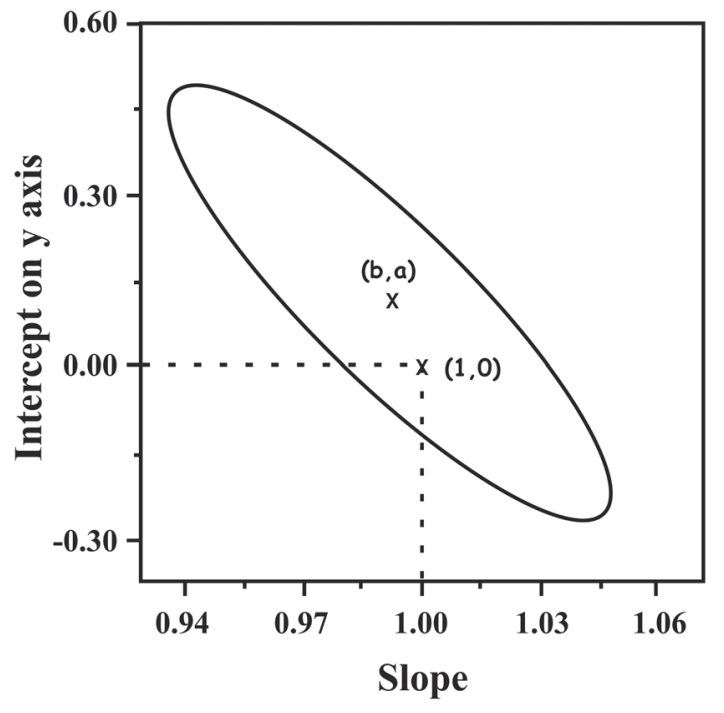

FIGURE 6 Comparison of the proposed method and a fluorometric one found in the literature ${ }^{[34]}$ for the determination of DA in urine. DA, dopamine

means that the accuracy of the proposed method and the reference method was not significantly different.

\section{4 | CONCLUSIONS}

UCNP/DA-melanin hybrid system provided a practical platform for quantifying DA in urine. The resulting UCNP-DA particles were well dispersed, with $10 \mathrm{~nm}$ in size and stable as determined by luminescence spectroscopy, UV-Vis spectroscopy, and high-resolution transmission electron microscopy. Aggregation decreased RLS in the nanoparticles and afforded a limit of detection of 1.62 $\mu \mathrm{M}$. This allows DA in biological body fluids such as urine to be determined with high sensitivity and selectivity. The proposed approach may thus be useful to develop simple, inexpensive ultrasensitive methods for detecting DA in a wide range of nanotechnological and biosensing applications.

\section{ORCID}

José Antonio Murillo Pulgarín (D) https://orcid.org/00000001-7475-6909

Aurelia Alañón Molina (D) https://orcid.org/0000-0002-78923492

\section{REFERENCES}

[1] J. A. Gingrich, M. G. Caron, Annu Rev Neurosci 1993, 16, 299.

[2] N. X. Tritsch, B. I. Sabatini, Neuron 2012, 76, 33.

[3] M. Vandecasteele, J. Glowinski, J. M. Deniau, I. Venance, Proc Natl Acad Sci 2008, 105, 4904. 
[4] M. R. Wightman, L. J. May, A. C. Michael, Anal Chem 1988, 60, 769A.

[5] B. R. Li, Y. J. Hsieh, Y. X. Chen, Y. T. Chung, C. Y. Pan, Y. T. Chen, J Am Chem Soc 2013, 135, 16034.

[6] X. D. Zhang, X. K. Chen, S. Q. Kai, H. Y. Wang, J. J. Yang, F. G. Wu, Z. Chen, Anal Chem 2015, 87, 3360.

[7] M. A. Medina, J. L. Urdiales, C. Rodríguez-Caso, F. J. Ramírez, F. Sánchez-Jiménez, Crit Rev Biochem Mol Biol 2003, 38, 23.

[8] K. Jackowska, P. Krisinski, Anal Bioanl Chem 2013, 405, 3753.

[9] L. A. Mercante, A. Pavinatto, L. E. O. Iwaki, V. P. Scagion, V. Zucolotto, O. N. Oliveira Jr., L. H. C. Mattoso, D. S. Correa, Appl Mater Interface 2015, 7, 4784.

[10] F. Malem, D. Mandler, Anal Chem 1993, 65, 37.

[11] W. C. Wu, H. W. Chang, Y. C. Tsai, Chem Commun 2011, 47, 6458.

[12] Y. Tao, Y. H. Liu, J. S. Ren, X. G. Qu, Biosens Bioelectron 2013, $42,41$.

[13] R. P. H. Nikolajsen, A. M. Hansen, Anal Chim Acta 2001, 449, 1.

[14] Q. Xu, J. Yoon, Chem Commun 2011, 46, 12497.

[15] Q. Mu, H. Xu, Y. Li, S. Ma, X. Zhong, Analyst 2014, 139, 93.

[16] M. R. Hormozi-Nezhad, A. Moslehipour, A. Bigdeli, Sens Actuators B 2017, 243, 715.

[17] W. Feng, C. Han, F. Li, Adv Mater 2013, 25, 5287.

[18] P. Qui, N. Zhou, H. Chen, C. Zhang, G. Gao, D. Cui, Nanoscale 2013, 5, 11512.

[19] R. F. Pasternack, C. Bustamante, R. F. Collings, A. Giannetto, E. J. Gibbs, JAm Chem Soc 1993, 115, 5393.

[20] C. Wang, C. Wang, Q. Wang, D. Chen, Anal Bioanal Chem 2017, 409, 2839.

[21] Q. Wei, H. Zhang, B. Du, Y. Li X., X. Zhang, Microchemica Acta 2005, 151, 59.

[22] Z. Chen, W. Ding, Y. Liang, F. L. Ren, Y. Han, J. Liu, Microchimica Acta 2005, 150, 35.
[23] Y. Kuang, S. Chen, Y. Long, Anal Bioanal Chem 2017, 409, 4001.

[24] J. A. Murillo, A. Alañón, Comput Chem 1993, 17, 341.

[25] Y. H. Wang, I. Bao, Z. H. Liu, D. W. Pang, Anal Chem 2011, 83, 8130.

[26] Y. H. Wang, R. X. Cai, Z. H. Liu, CrysEngComm 2011, 13, 1772.

[27] G. Swan, Ann N Y Acad Sci 1963, 100, 1005.

[28] P. J. Rousseeuw, P.J., A. M. Leroy, Robust Regression and Outlier Detection, Wiley, New York 1987.

[29] P. D. Lark, B. R. Craven, R. C. L. Bosworth, The Handling of Chemical Data, Pergamon, Exeter 1968.

[30] D. L. Massart, B. G. M. Vandeginste, S. N. Deming, L. Kaufman, Chemometrics: a Textbook, Elsevier, Oxford 1968.

[31] J. N. Miller, Analyst 1991, 116, 3.

[32] G. Long, J. D. Winefordner, Anal Chem 1983, 55, 712A.

[33] H. Y. Wang, M. Zhang, H. Zhao, Anal Sci 2007, 23, 1297.

[34] H. Y. Wang, Q. S. Hui, L. X. Xu, J. G. Jiang, Y Sun Anal Chim Acta 2003, 497, 93.

\section{SUPPORTING INFORMATION}

Additional supporting information may be found online in the Supporting Information section at the end of the article.

How to cite this article: Pulgarín JAM, Molina AA, García EJ, Gómez LG. A sensitive resonance Rayleigh scattering sensor for dopamine in urine using upconversion nanoparticles. J Raman Spectrosc. 2019;1-8. https://doi.org/10.1002/jrs.5800 